\title{
DETERMINATION OF ILLUMINANTS REPRESENTING TYPICAL WHITE LIGHT EMITTING DIODES SOURCES
}

\author{
Jost, S. ${ }^{1}$, Ngo, M. ${ }^{1}$, Ferrero, A. ${ }^{2}$, Poikonen, T. ${ }^{3}$, Pulli, T. ${ }^{4}$, Thorseth, A. ${ }^{5}$, Blattner, P. ${ }^{6}$ \\ 1 Univ Lyon, ENTPE, LGCB, Vaulx-en-Velin, FRANCE, \\ 2 Instituto de Óptica, CSIC, Madrid, SPAIN \\ 3 VTT, Ltd, Espoo, FINLAND \\ ${ }^{4}$ Aalto, Espoo, FINLAND \\ ${ }^{5}$ DTU Fotonik, Roskilde, DENMARK \\ ${ }^{6}$ METAS, Bern-Wabern, SWITZERLAND \\ sophie.jost@entpe.fr
}

\begin{abstract}
Solid-state lighting (SSL) products are already in use by consumers and are rapidly gaining the lighting market. Especially, white Light Emitting Diode (LED) sources are replacing banned incandescent lamps and other lighting technologies in most general lighting applications. The aim of this work is to develop LED-based illuminants that describe typical white LED products based on their Spectral Power Distributions (SPDs). Some of these new illuminants will be recommended in the update of the CIE publication 15 on colorimetry with the other typical illuminants, and among them, some could be used to complement the CIE standard illuminant $A$ for calibration use in photometry.
\end{abstract}

Keywords: Solid-State Light Source, Light Emitting Diode, Illuminant, Photometry, Colorimetry.

\section{Introduction}

Solid-state lighting (SSL) products are already in use by consumers and are rapidly gaining the lighting market. Especially, white Light Emitting Diode (LED) sources are replacing banned incandescent lamps and other lighting technologies in most general lighting applications. Spectral Power Distributions (SPDs) of LEDs are quite different from those of conventional light sources, which raises questions about applicability of colorimetry and photometry based on the Standard Illuminant A.

Currently, most lighting products measured in laboratories are LED sources while incandescent lamps are still the standard sources used in photometric calibrations. However, in order to keep spectral mismatch errors of the measurement small, it is important to use a calibration source with a SPD close to the SPD of the tested light.

The new three-year European research project EMPIR 15SIB07 PhotoLED (Future Photometry Based on Solid State Lighting Products), aims to solve these issues by selecting new calibration spectra and developing standard lamps based on white LED sources in close connections with CIE Division 1 and Division 2.

The aim of this work is to develop LED-based illuminants that describe typical white LED products based on their SPDs. Some of these new illuminants will be recommended in the update of the CIE publication 15:2004 (CIE, 2004) on colorimetry with the other typical illuminants and among them, some could be used to complement CIE Standard Illuminant A for calibration use in photometry.

This study summarizes the sorting of the data according to different spectral shapes and Correlated Colour Temperatures (CCTs) and the analysis proceeded to select representative illuminants for white SSL products.

\section{Method}

This part summarizes the process of selection of representative illuminants for white LEDs spectra. Several methods based on a closeness score were explored in order to identify representative LED SPDs for different CCTs. 


\subsection{Collection, normalization and categorization}

\subsubsection{Database}

During autumn 2016, 1298 SPDs of white LED sources available on the market were collected from the partners and collaborators of the project PhotoLED. Only spectral data measured with traceable methods were used (relative radiant flux in sphere or irradiance with goniometer or bench). The spectral data were collected into a database of typical white LED SPDs covering the spectral range from $380 \mathrm{~nm}$ to $780 \mathrm{~nm}$ with $1 \mathrm{~nm}$ step.

In order to represent them and to accommodate the SPDs for the selection procedure, we normalized the data at the same $\mathrm{Y}$ value:

$$
S_{z}(\lambda)=\frac{S(\lambda)}{\int_{380}^{780} S(\lambda) \cdot V(\lambda) \cdot d \lambda}
$$

\subsubsection{CCT bin categorization and outliers}

The spectral data were distributed into CCT bins based on ANSI Standard C78.377.2011 (ANSI, 2011). The specifications for the chromaticity of Solid State Lighting (SSL) products, provided by ANSI, were also applied to extract outlier SPDs. A total of eight different CCT bins $(2700 \mathrm{~K}, 3000 \mathrm{~K}, 3500 \mathrm{~K}, 4000 \mathrm{~K}, 4500 \mathrm{~K}, 5000 \mathrm{~K}, 5700 \mathrm{~K}, 6500 \mathrm{~K})$ were obtained. Figure 1 represents the repartition of the chromaticities in CIE 1931 chromaticity diagram for the eight CCT bins. The red points represent the 176 outliers that were taken out of the original database for the analysis. Three additional SPDs were also excluded from the analysis because of aberrant measurements in higher wavelengths.

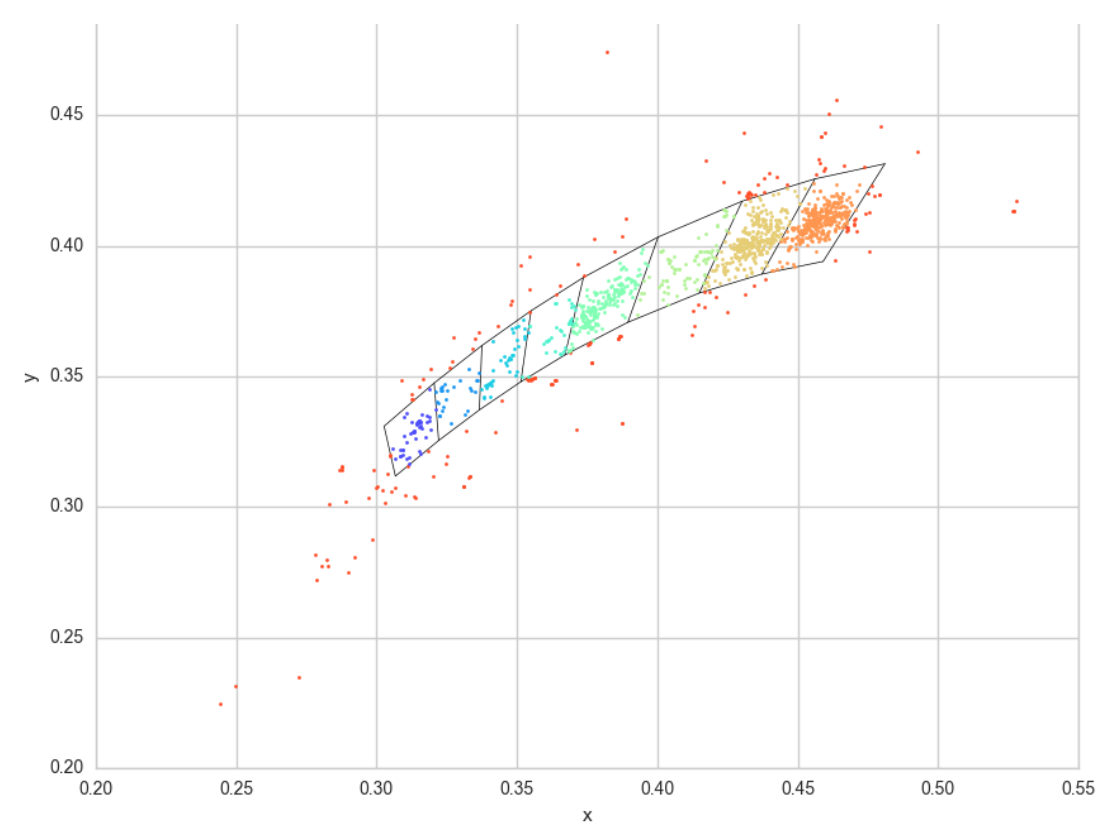

Figure 1 - Chromaticity of the LED spectra within the database

\subsubsection{Typical white LED technologies}

The typical white LED technologies of the remaining SPDs in the database were identified. Within this database there were mainly phosphor-converted blue LEDs (BLED - 1068 SPDs), then hybrid LEDs which consist in a mixing of phosphor-converted blue LED and red LED (45), four phosphor-converted violet LEDs (VLEDs) and two RGB LEDs composed of mixing of red, green and blue LEDs. 


\subsection{Determination of the centroid SPDs}

\subsubsection{Definition of a closeness score}

To determine the centroid SPD of each category (bin or technology) the method was to find the closest SPD to all other SPDs within its category. To do so, a closeness score was defined and calculated for each SPD as:

$$
C_{i}^{b}\left(S_{z i}(\lambda)\right)=\sum_{k \in b} 10^{-d\left(S_{z i}(\lambda) S_{z k}(\lambda)\right)}
$$

where $b$ represents the category, $S_{z i}$ is the $i^{\text {th }}$ SPD of the category $b$ and $d$ is any mathematical distance function.

This closeness score quantifies with a single number the similarity of each SPD to the other SPDs in the category. The centroid SPD for each category is the SPD which obtains the highest score.

Note that the distance $d$ can be computed in various ways, e.g.:

- 1-Norm distance defined as $d_{1}\left(S_{z 1}(\lambda), S_{z 2}(\lambda)\right)=\sum_{\lambda=350}^{850}\left|S_{z 1}(\lambda)-S_{z 2}(\lambda)\right|$

- 2-Norm or Euclidean distance defined as $d_{2}\left(S_{z 1}(\lambda), S_{z 2}(\lambda)\right)=\sum_{\lambda=350}^{850} \sqrt{\left(S_{z 1}(\lambda)-S_{z 2}(\lambda)\right)^{2}}$

- Etc.

\subsubsection{Choice of distance}

We tested 1-Norm and Euclidean distances and as expected the choice of distance impacts the determination of the centroid. Figure 2 -shows an example for the $4500 \mathrm{~K} \mathrm{CCT}$ bins. Because the Euclidean distance favors smoother shapes (the RMS function tends to reduce the impact of higher individual features), we decided to use this distance for the analysis.

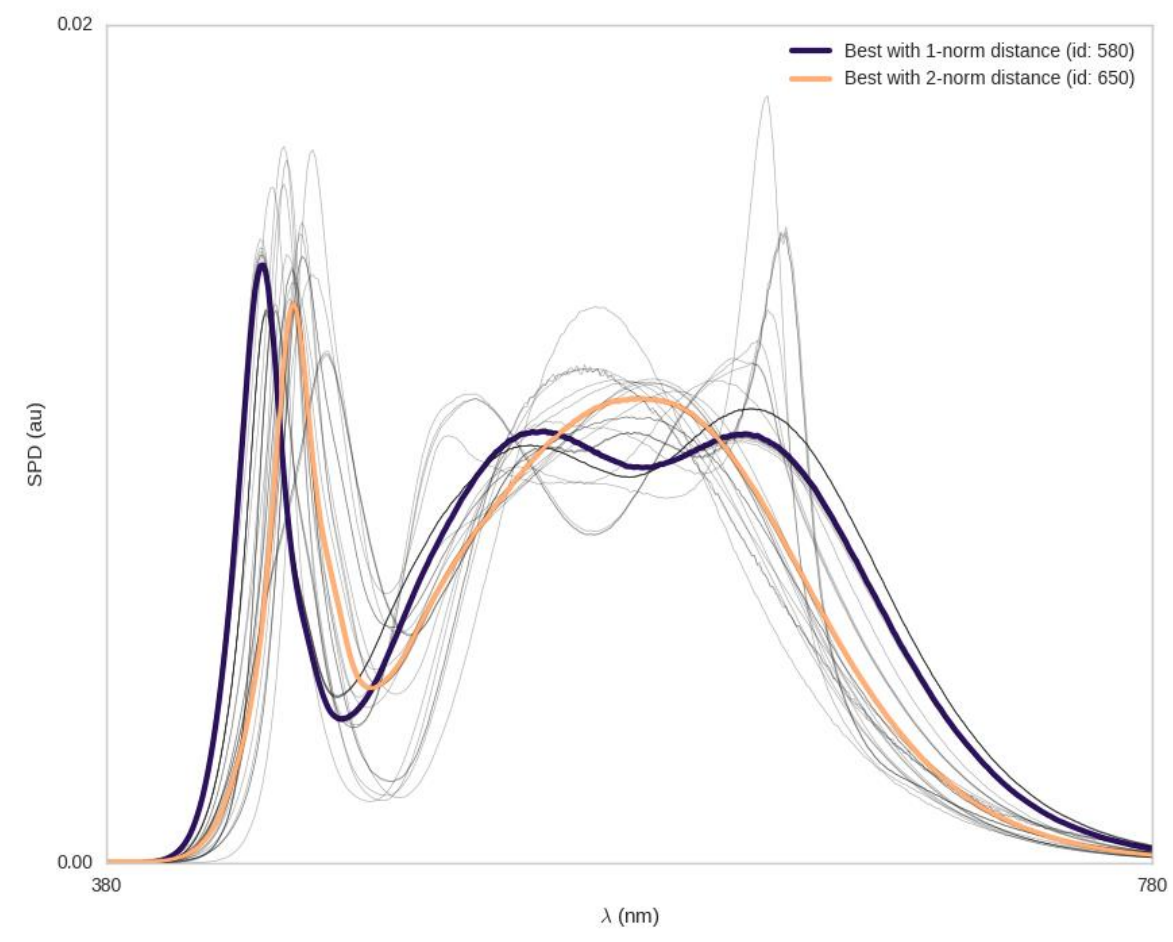

Figure 2 - Centroid SPDs for the $4500 \mathrm{~K}$ bin (Blue - 1-Norm distance; Red - 2-Norm distance)

\subsection{Determination of an averaged SPD different from the closest one}

In order to avoid the issue of recommending a representative LED illuminant of one particular LED manufacturer but to determine a SPD with feasible spectral features, different methods 
of averaging were investigated to define a representative illuminant close but different of the centroid:

- Averaging the SPDs of $n$ SPDs with the highest closeness scores;

- Averaging the SPDs with a closeness score above a fixed threshold;

- Averaging the SPDs with a closeness score above a sliding threshold;

- Averaging the SPDs of the best closeness scores and the $\mathrm{n}$ closest to it.

The first three methods lead to averaged SPDs with shapes that might be hard to reproduce, therefore the last averaging method was applied to the SPD with the highest closeness score and the nine SPDs closer to it. Figure 3 represents for the eight CCT bins the centroid SPD (dotted line) and the averaged SPD (full line).

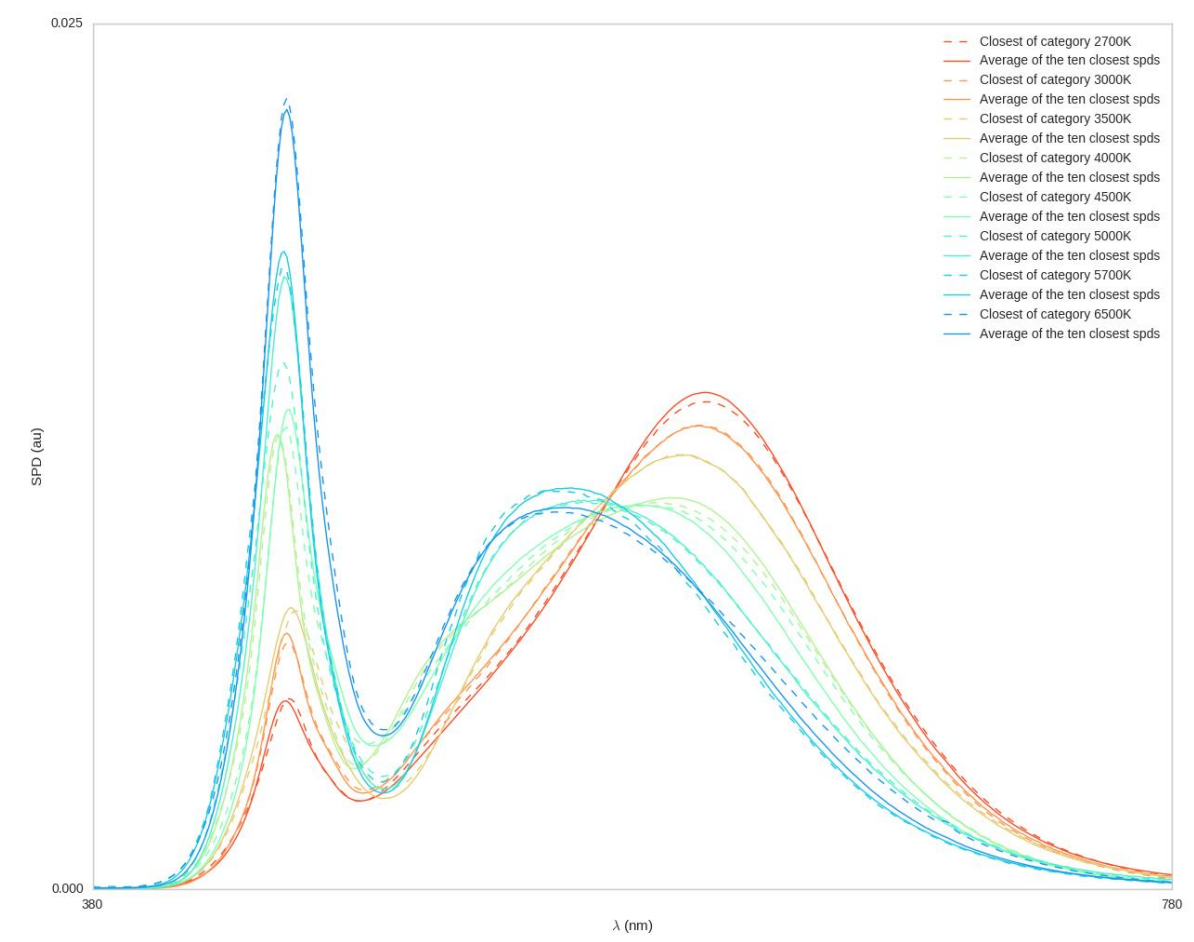

Figure 3 - Centroid (dotted line) and averaged SPDs (full line) for the eight CCT bins

\subsection{Determination of representative illuminants}

The final step for determining the representative illuminants was to clean the data of measurements artifacts. To avoid ripples due to measurement noise, the data is first smoothed out. Then, some of the spectral data used in for determining the representative spectra were most likely measured using a single grating spectrometer, resulting in measurement issues due to straylight. To contravene those, an extrapolation method based on the one proposed in IES TM-30-15 Annex 1 (IES, 2015) was applied.

\section{Results}

\subsection{Summary of the method}

The final process to determine the illuminants representing typical white LED spectra was:

1. Normalizing each SPD at the same $Y$ values.

2. Computing a closeness score for each SPD within its category with a Euclidean distance.

3. Determining the closest SPD for each category (centroid SPD).

4. Determining a set of 10 SPDs for each category, with the centroid SPD and its nine closest SPDs. 
5. Averaging those SPDs (averaged SPD).

6. Smoothing the averaged SPDs and extrapolating the smoothed SPDs (typical illuminant).

\subsection{Representative Illuminants for phosphor-converted blue LEDs}

Following this method, eight representative illuminants (one for each CCT bin) have been determined for phosphor-converted blue LEDs (Figure 4). Note that the representative SPDs obtained are the same when applying the method to the whole database (without outliers) or to BLEDs only.

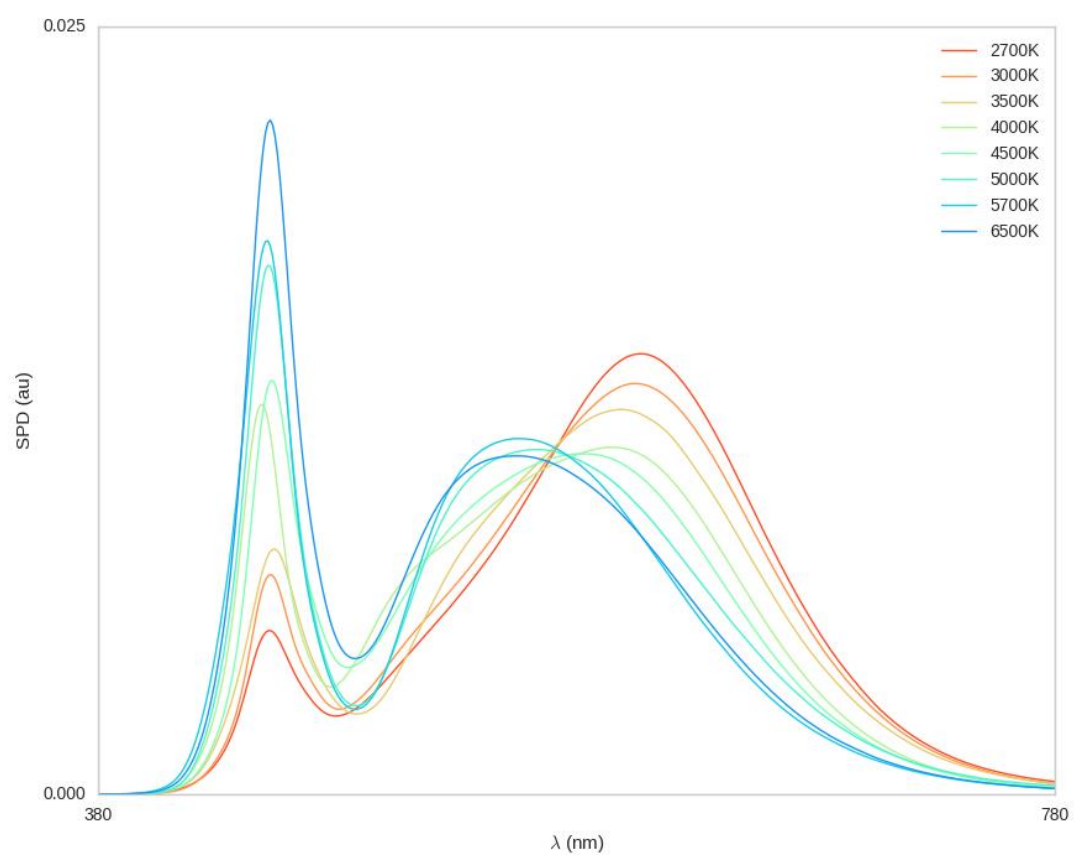

Figure 4 - Representative illuminants for phosphor-converted blue LEDs for the eight CCT bins

\subsection{Representative Illuminants for hybrid LEDs}

Due to the number of hybrid LEDs in the database, the same method was applied to them SPDs regardless of the CCT. Thus one illuminant representing typical hybrid LED spectrum has been determined (Figure 5).

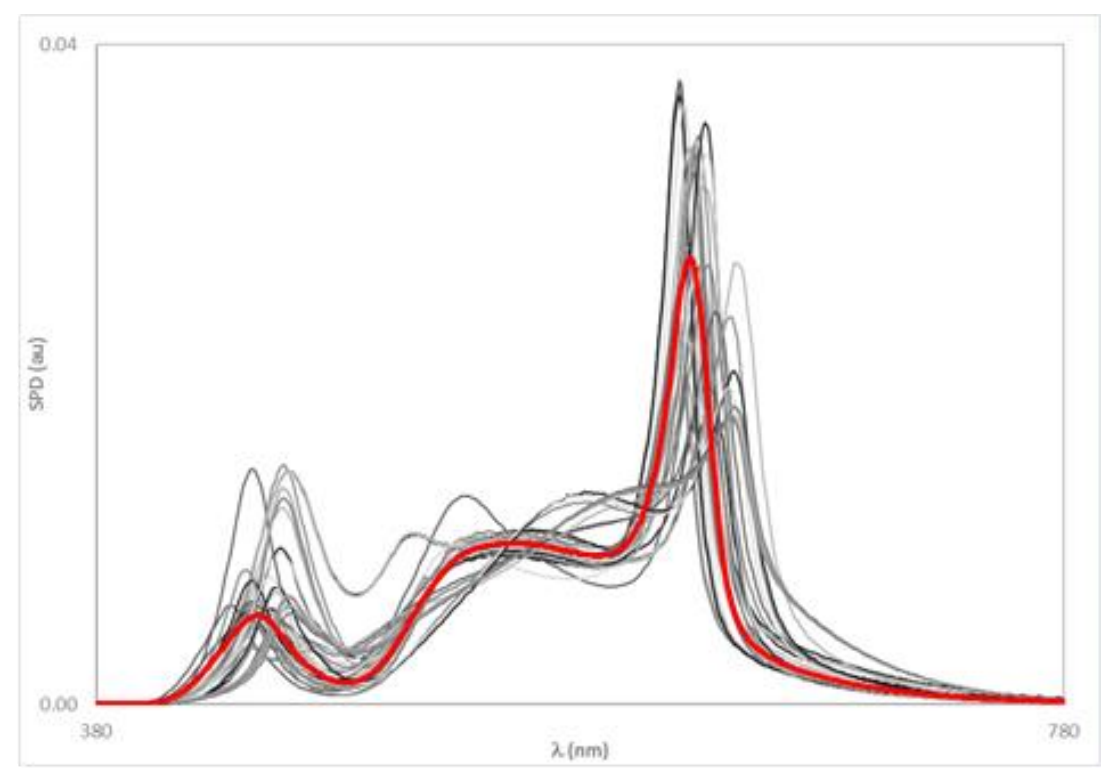

Figure 5 - The 45 hybrid LEDs with their representative SPD (in bold red) 


\subsection{Other typical shape of LEDs}

VLEDs and RGB LEDs were not numerous enough to extract typical representative illuminants.

\section{Conclusion}

The EMPIR project PhotoLED is working towards a new system of photometry based on white LED sources. A large database of collected traceable relative spectral data of white LED products was analysed to be used in applications of colorimetry and photometry.

At the time of writing this article, information about Division 1 possibly adopting many of the representative spectra was received. They might be published in the new version of $\mathrm{CIE}$ Publication 15 on colorimetry (work of TC1-85) as illuminants representing typical LED lamps as it was done previously for fluorescent or HID lamps. Some BLED SPDs most representative of the widespread CCTs and the hybrid LED SPD are under consideration. Although not representative of the market, the two RGB LEDs and two violet LEDs might also be included in the publication to illustrate the spectral shapes of these types of LED sources. The new representative spectra proposed for colorimetry will also form the basis of discussion to choose appropriate LED calibration spectra for photometry.

\section{Acknowledgments}

The work leading to this study is partly funded by the European Metrology Programme for Innovation and Research (EMPIR) Project 15SIB07 PhotoLED "Future Photometry Based on Solid State Lighting Products". The EMPIR initiative is co-funded by the European Union's Horizon 2020 research and innovation programme and the EMPIR Participating States. The authors would like to thank all partners and collaborators of the project who provided spectral data of LED products for the analysis.

\section{References}

ANSI C78.377.2011. American National Standard. Specifications for the Chromaticity of Solid State Lighting Products. Publication ANSI_NEMA_ANSLG C78.377-2008. Rosslyn (VA): American National Standard Lighting Group. C̄78.377.2011.

CIE 2004. CIE15:2004 Colorimetry. Vienna: CIE.

IES TM-30-15. IES method for evaluating light source color rendition. 